ference for "unfolded" proteins. J. Brandts (University of Massachusetts) discussed the unfolding of ribonuclease in terms of the possible intermediate states which are clearly indicated by kinetic measurements, although the thermodynamics are compatible with a two-state model, implying that all steps bar one are associated with zero enthalpy changes. This condition is met by the cis-trans isomerisation of proline which may therefore account for the rate-determining step, whereas conformational changes are associated with relaxation times of $1-100 \mathrm{~ms}$. The folding/unfolding equilibria of all small proteins appear to be consistent with a rate-determining step corresponding to a cis-trans isomerisation.

A description of the dynamic nature of folded proteins presents many topical problems and both $\mathbf{R}$. Lumry and A. Rosenberg (University of Minnesota) stressed the importance of high frequency $\left(10^{8} \mathrm{~s}^{-1}\right)$ conformational fluctuations to the catalytic functions of proteins. The existence of free volume fluctuations is consistent with the high diffusion rates of large molecules (such as acrylonitrile) in folded proteins and with the marked pressure dependence of proton exchange rates which are not observed with homopolypeptides or oxidised ribonuclease:

A thermodynamic characterisation of biochemical processes by itself is not always as interesting as an insight into molecular mechanisms, and this implicitly takes us into the realm of statistical thermodynamics. F. Franks (Unilever Research, Sharnbrook) discussed the combination of thermodynamic data and computational methods aimed at the derivation of a potential function for the hydrophobic interaction. This in turn allows the calculation of the radial distribution function and hence the thermodynamic properties associated with the interaction of apolar particles in water. In a complementary paper, E. Clementi (Istituto Donegani, Novara) outlined a combination of $a b$ initio and computer simulation approaches to the study of amino acid hydration.

\section{Exploding black holes}

\author{
from P. C. W. Davies
}

A symposium organised by $R$. Penrose and D. Sciama was held in Oxford on April 20 and 21, 1976.

Black holes are either a French, German or American idea, according to opinion. But if they explode, they are British. At an historic Oxford sympo- sium early in 1974, Stephen Hawking of the University of Cambridge, revealed an astonishing theoretical disccvery-black holes are not black after all, but radiate with a characteristic blackbody (thermal equilibrium) spectrum, at a temperature which increases as the mass dwindles, ensuring the eventual demise of these objects in a sudden explosion.

Since then, the analysis of what is now referred to as the Hawking process has become a veritable industry in South East England, with no less than three major relativity groups (Cambridge, London and Oxford) actively studying the details and implications of the new discovery. Hawking's prediction itself was the result of applying quantum theory to black holes. The remarkable conclusion is that the violent distortion of space-time in their vicinity leads to the production of radiation with a definite temperature. The significance of this lies not so much in observation (the temperature of a solar mass black hole is a mere $10^{-6} \mathrm{~K}$ ) but in establishing important theoretical connections hitherto barely suspected.

It had previously been noted by some relativists that black hole processes bear a superficial resemblance to thermodynamics. As long as the treatment remained classical the resemblance was only an analogy. The key step, which Hawking took, was to apply quantum mechanics. The result has been to provide an amazing underlying connection between quantum theory, general relativity and thermodynamics.

As experts in any of these three disciplines tend to be lamentably ignorant of the other two, it was particularly appropriate that this year's Oxford symposium entailed a programme of lectures and reviews by practitioners of each subject. The conference provided an opportunity for an unusual interplay of ideas, with the Hawking process as a central theme.

Hawking himself delivered some provocative and fascinating extensions of his original idea, by dealing with the conceptual and philosophical aspects of particle production effects. He and G. Gibbons (University of Cambridge) presented arguments in support of the proposal that event horizons cause radiation production under quite general circumstances. In particular, the claim that particle detectors in de Sitter space would also detect radiation seems to be a direct challenge to the conventional understanding of some fundamental aspects of relativity and quantum theory. This challenge was taken up by some of the other participants in fruitful but inconclusive exchanges. Controversy centred around Hawking's claim that much of physics is observer-dependent. Two observers in different states of motion might encounter two entirely dissimilar histories of the Universe, he alleges. In particular, the traditional theoretical procedure of attempting to construct a covariant energy-momentum tensor which is independent of observer is misguided. Ironically, this procedure is precisely what several research groups have been trying to do, with a view to studying how quantum effects react back on the geometry of space-time. According to Hawking, the traditional energy-momentum tensor calculations are wrong. Indeed, they contain socalled vacuum polarisation terms, which he said he did not believe in.

It will take some time for the full implications of these intriguing new ideas to sink in. The more traditional approach was defended by Bryce DeWitt (at present at the University of Oxford). He described some of the conventional energy-momentum tensor calculations, and also presented an interesting model of a particle detector which appeared to conflict with Hawking's radical departure.

Much of the remainder of the conference was devoted to exposition about some of the peripheral aspects of the Hawking process. Some of the review talks were interesting and entertaining in their own right, particularly that of Donald Lyden-Bell (University of Cambridge) who presented a brilliant review of the thermodynamics of classical self-gravitating systems. Other speakers, such as Jergen Ehlers (Munich) who spoke about relativistic kinetic theory and statistical mechanics, and Bernard Schutz (Cardiff), who dealt with stability in relativity, clearly touched on subject matter which will eventually be relevant to the hoped-for unification of quantum field theory, thermodynamics and gravitation. But at this stage only a jumble of halfconnected ideas has emerged. Other speakers were John Steward (Cambridge), Robert Thouless (Birmingham) and Oliver Penrose (Open University).

The cross-fertilisation between these subject areas is clearly of great importance in stimulating further research in this new subject. The conference organisers, Roger Penrose and Dennis Sciama, both gave thought-provoking general assessments, tying the strands of the separate topics together. It is hard to resist their own impression that much more lies beneath the surface; many disconnected ideas seem to be associated in some, as yet vague, but intriguing fashion. In this fastmoving field of research, it is probable that another Oxford symposium in the near future will provide still more fundamentally new results, which could well have implications far beyond the specialist topic of black holes. 